\title{
Dark matter search results from DEAP-3600 at SNOLAB
}

\author{
Simon Viel ${ }^{a, b, *}$ for the DEAP-3600 Collaboration ${ }^{\dagger}$ \\ ${ }^{a}$ Department of Physics, Carleton University, Ottawa, Ontario, K1S 5B6, Canada \\ ${ }^{b}$ Arthur B. McDonald Canadian Astroparticle Research Institute, Queen's University, Kingston, Ontario, \\ K7L 3N6, Canada
}

E-mail: simon.viel@snolab.ca

The latest physics results from DEAP-3600 are summarized. DEAP-3600 is searching for dark matter interactions with a liquid argon target, shielded from cosmic rays by over $2 \mathrm{~km}$ of rock at SNOLAB in Sudbury, Canada. The spherical detector consists of 3.3 tonnes of liquid argon in a large ultralow-background acrylic vessel instrumented with 255 photomultiplier tubes. DEAP-3600 is sensitive to nuclear recoils from dark matter particles, which would cause the emission of prompt scintillation light. Backgrounds come from alpha particles on the inner detector surfaces, from external neutrons, from ${ }^{39} \mathrm{Ar}$ beta decays, and from trace radioactivity in detector components. DEAP-3600 demonstrates excellent performance for pulse-shape discrimination, event reconstruction, background rejection and sensitivity to dark matter.

40th International Conference on High Energy physics - ICHEP2020

July 28 - August 6, 2020

Prague, Czech Republic (virtual meeting)

\footnotetext{
${ }^{\dagger}$ We thank the Natural Sciences and Engineering Research Council of Canada, the Canadian Foundation for Innovation (CFI), the Ontario Ministry of Research and Innovation (MRI), and Alberta Advanced Education and Technology (ASRIP), Queen's University, the University of Alberta, Carleton University, the Canada First Research Excellence Fund, the Arthur B. McDonald Canadian Astroparticle Research Institute, DGAPA-UNAM (PAPIIT No. IN108020) and Consejo Nacional de Ciencia y Tecnología (CONACyT, Mexico, Grant A1-S-8960), the European Research Council Project (ERC StG 279980), the UK Science and Technology Facilities Council (STFC) (ST/K002570/1 and ST/R002908/1), the Russian Science Foundation (Grant No. 16-12-10369), the Leverhulme Trust (ECF-20130496), the Spanish Ministry of Science, Innovation and Universities (FPA2017-82647-P grant and MDM-2015-0509), and the International Research Agenda Programme AstroCeNT (MAB/2018/7) funded by the Foundation for Polish Science (FNP) from the European Regional Development Fund. Studentship support from the Rutherford Appleton Laboratory Particle Physics Division, STFC and SEPNet PhD is acknowledged. We thank SNOLAB and its staff for support through underground space, logistical, and technical services. SNOLAB operations are supported by the CFI and Province of Ontario MRI, with underground access provided by Vale at the Creighton mine site. We thank Vale for their continuing support, including the work of shipping the acrylic vessel underground. We gratefully acknowledge the support of Compute Canada, Calcul Québec, the Centre for Advanced Computing at Queen's University, and the Computation Centre for Particle and Astrophysics (C2PAP) at the Leibniz Supercomputer Centre (LRZ) for providing the computing resources required to do this work.

${ }^{*}$ Speaker
} 


\section{Introduction}

Dark matter direct detection experiments look for new particles beyond the Standard Model of particle physics, that could create observable signals when they interact with ordinary matter in the detector's target. In particular, Weakly Interacting Massive Particles (WIMPs) are hypothesized to be electrically neutral particles with masses in the approximate range $1 \mathrm{GeV} / \mathrm{c}^{2}$ to $100 \mathrm{TeV} / \mathrm{c}^{2}$ and interaction coupling strengths with ordinary matter that are below the weak interaction coupling of the Standard Model. Dark matter comprises approximately 27\% of the total mass-energy density of the Universe, while ordinary matter only accounts for 5\% of this total [1].

DEAP-3600 is located at SNOLAB, Canada's deepest underground laboratory in the Vale Creighton mine, $2070 \mathrm{~m}$ below Sudbury, Ontario. At the heart of this experiment is a $1.7 \mathrm{~m}$ diameter acrylic vessel containing 3.3 tonnes of liquid argon (LAr) instrumented with 255 photomultiplier tubes (PMTs). Its objective is to detect dark matter particles coming into the LAr volume where they may collide with an argon nucleus, causing a nuclear recoil that excites and ionizes argon atoms along its path. This most frequently results in the formation of excited argon dimers, and their following de-excitation via $\mathrm{Ar}_{2}^{*} \longrightarrow 2 \mathrm{Ar}+h v(128 \mathrm{~nm})$. This ultraviolet scintillation light propagates to the inner surface of the acrylic vessel, where it is wavelength-shifted to visible by a layer of tetraphenyl butadiene (TPB). Visible photons then enter the acrylic light guides and are collected by the PMTs. Details on the design and construction of DEAP-3600 are given in [2].

The most recent physics results from DEAP-3600 are summarized here. They are based on the first year of our second-fill dataset, taken from November 2016 to October 2017. In particular, the main dark matter search results summarized here have been obtained with this exposure of $3279 \mathrm{~kg}$ LAr in 231 live-days [3], superseding our previous first-fill results with $3322 \mathrm{~kg}$ LAr in 4.4 live-days collected in August 2016 [4]. Analysis of the full second-fill dataset of DEAP-3600, collected up to March 28th, 2020, is in progress. These data were collected with a detector uptime of $97 \%, 88 \%$ of which constitute the dark matter search dataset with the remainder being radioactive source and light injection calibrations. Our physics data collection is 80\% blind since January 2018.

\section{Pulse-shape discrimination}

Crucial to the sensitivity of DEAP-3600 to nuclear recoils (NRs) from dark matter interactions is the rejection of electronic recoils (ERs), using a technique called pulse-shape discrimination (PSD). The LAr scintillation pulse-shape has been studied in detail in [5], with one of the main fit results shown here in Figure 1 (left). A short time constant $\sim 8 \mathrm{~ns}$ originates from the prompt de-excitation of the $\mathrm{Ar}_{2}^{*}$ dimer in the singlet state, combined with the effect of TPB prompt decay times and the time structure from photon propagation in the detector. In contrast, a long time constant $\sim 1.4 \mu$ s is due to the long-lived triplet state of $\mathrm{Ar}_{2}^{*}$, and is dependent on the LAr purity. At longer times, the average pulse-shape is dominated by PMT afterpulsing and the long decay times of TPB, before eventually coming down to a baseline of stray delayed light and PMT dark rates.

The power of PSD comes from the fact that NRs predominantly excite the singlet state of LAr, as opposed to the triplet state, with greater relative amplitude compared to ERs. This implies that LAr scintillation light originating from NRs is more prompt than LAr scintillation light originating from ERs [6]. In this analysis, the PSD variable $F_{\text {prompt }} \in[0,1]$ is defined as: 


$$
F_{\text {prompt }}=\frac{\sum_{t=-28 \mathrm{~ns}}^{60 \mathrm{~ns}} \mathrm{PE}(t)}{\sum_{t=-28 n s}^{10 \mu \mathrm{s}} \mathrm{PE}(t)}
$$

counting the fraction of photoelectrons (PE) detected in a prompt window around the event time, over the total number of PE detected, factoring out charge produced by afterpulsing. Figure 1 (right) shows this variable in AmBe neutron source calibration data. Signal-like NRs from neutron single-scatter events peak at $F_{\text {prompt }} \sim 0.7$, clearly separated from the ER background events consisting mainly of ${ }^{39} \mathrm{Ar} \beta$ decays. DEAP-3600 demonstrated world-leading PSD performance, with a remarkably low ER leakage probability of $4.1_{-1.0}^{+2.1} \times 10^{-9}$ with $90 \%$ NR acceptance in the dark matter search region of 15.6-32.9 $\mathrm{keV}_{\mathrm{ee}}$ [3].
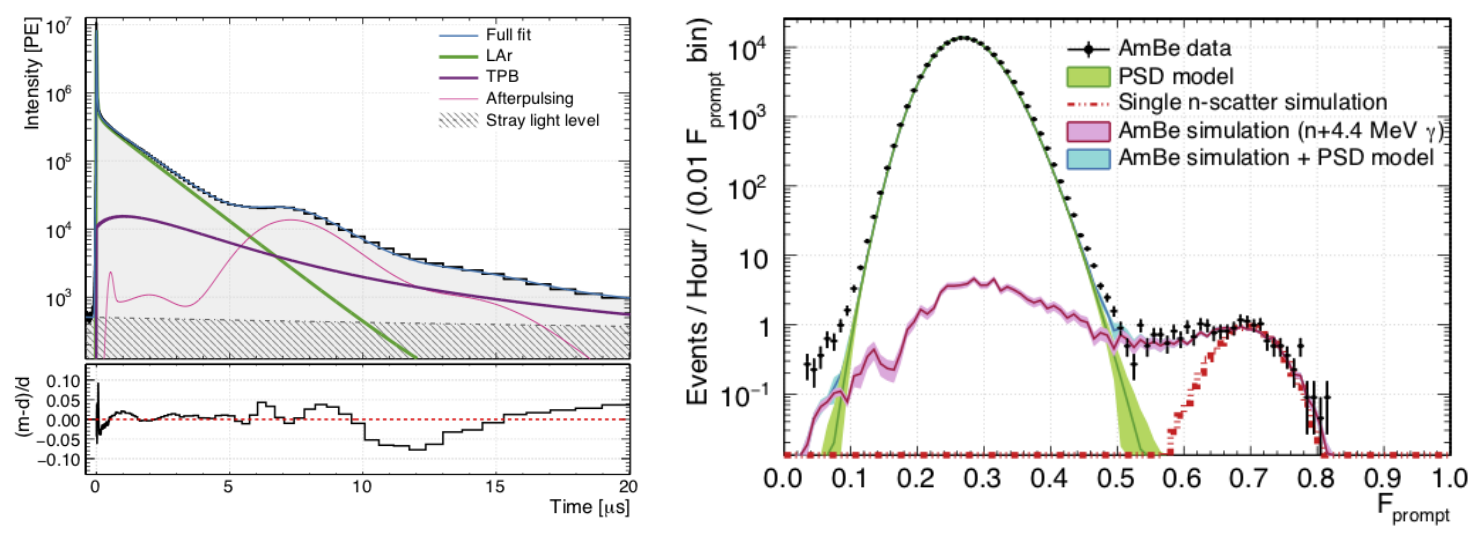

Figure 1: (Left) LAr scintillation pulseshape from ${ }^{39} \mathrm{Ar} \beta$ decays in DEAP-3600, from approximately 200,000 events in the range 100-300 PE, featuring good agreement with the pulseshape model [5]. (Right) Distribution of $F_{\text {prompt }}$ in AmBe neutron source calibration data, in the range 120-200 PE [3].

\section{Background model}

Electromagnetic backgrounds to the dark matter search mainly come from the ${ }^{39} \mathrm{Ar} \beta$ decays discussed above. A full characterization of ERs induced by $\beta$ and $\gamma$ rays from internal and external radioactivity in DEAP-3600 has been published in [7]. The ER background model is in good agreement with data over 9 orders of magnitude in event rate, up to at least $5 \mathrm{MeV}$ in energy. This analysis also measured the specific activity of ${ }^{42} \mathrm{Ar}$ in the atmosphere, by measuring the daughter decay of ${ }^{42} \mathrm{~K}$ in our detector: the result is $40.4 \pm 5.9 \mu \mathrm{Bq} /(\mathrm{kg}$ of atmospheric argon) [7].

Neutrons are a background to the dark matter search when they cause single-scatter NR events in the LAr. Events consistent with multiple scattering are rejected in the analysis. The remaining background rate is estimated using a dedicated data control region that takes advantage of $\gamma$-rays from neutron capture on ${ }^{1} \mathrm{H}$ or on ${ }^{40} \mathrm{Ar}$ detected in a $1 \mathrm{~ms}$ coincidence window following the NR.

Alpha backgrounds in DEAP-3600 come in at least three event types: (1) $\alpha$ decays in the LAr bulk, (2) $\alpha$ decays on the inner acrylic vessel surface, (3) $\alpha$ decays on the acrylic neck flow-guides at the top of the vessel. In the case of $\alpha$ decays in the LAr bulk, the full $\alpha$ energy is deposited in LAr, which results in two orders of magnitude more scintillation light produced compared to possible dark matter interactions. 
In contrast, events caused by $\alpha$ decays on the acrylic vessel surface can leak into the energy region of interest for WIMP dark matter, if most of the surface $\alpha$ energy is deposited in the acrylic. These surface events are rejected with position reconstruction, by selecting a fiducial volume in the centre of the detector for the dark matter search. Two algorithms are employed for this purpose: a "PE-based" algorithm using the full $10 \mu$ s event window (PMTs closer to the event detect a higher fraction of the PE) and a new "time-based" algorithm using only the first $40 \mathrm{~ns}$ of the event (PMTs closer to the event detect earlier photon arrival times) [8]. Both the surface and LAr bulk $\alpha$ decays are very well-described by our background model, demonstrating the very low levels of radon contamination that were achieved during the construction of DEAP-3600 [3].

A particularly challenging background comes from $\alpha$ decays occurring on the neck of the detector. The geometry of the neck flow-guides can result in shadowing of scintillation light, such that only a small fraction of the photons from the event are detected by the PMTs. This can result in a signal-like event, with a reconstructed position in the fiducial region. Fortunately, as seen in Figure 2, features are visible in reconstructed position distributions as a function of PE detected, that match the correlations observed in Monte Carlo simulations of $\alpha$ decays on the neck flow-guide surfaces. Such features allow a template fit using multiple control regions (CRs), that results in the determination of neck $\alpha$ background event rates.
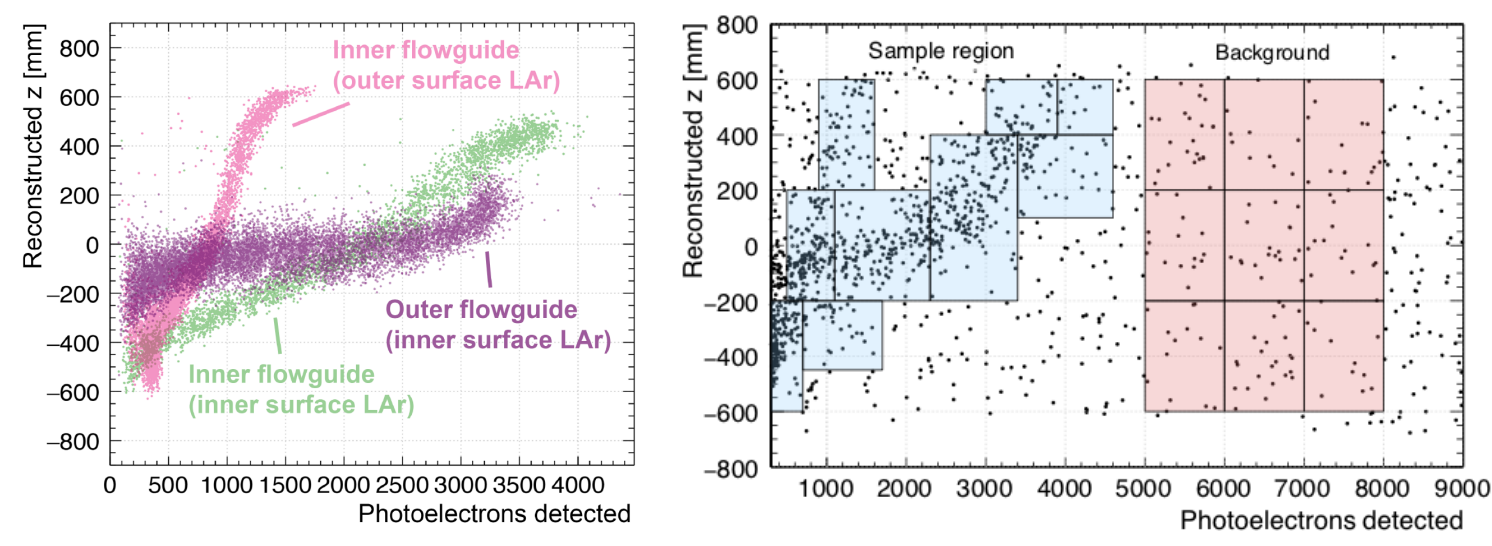

Figure 2: (Left) In simulated neck $\alpha$ events, clear correlations are seen between the reconstructed vertical position $z$ and the number of PE detected. (Right) These same features are seen in data, allowing the definition of CRs. The rate from an additional source of background events is subtracted from the neck $\alpha$ CRs. [3]

Owing to the unique characteristics of neck $\alpha$ events, a dedicated selection is used to reject this background. In contrast to signal, these events more frequently result in excess light in the top rows of PMTs, or early light in PMTs above the LAr fill level. Two bundles of wavelength-shifting optical fibres wrapped around the detector neck are instrumented with dedicated PMTs at both ends: events are vetoed when these PMTs detect light. In neck $\alpha$ events, the PE-based and time-based reconstructed positions tend to differ, while the two algorithms agree when the event genuinely comes from the LAr bulk [8]. Finally, PSD is also useful against neck $\alpha$ events: whereas higherenergy $\alpha$ decays have slightly higher $F_{\text {prompt }}$ compared to low-energy NRs expected from WIMPs, neck $\alpha$ events are actually higher-energy decays that only appear at lower detected PE because of the flow-guide shadowing effect, so they too have higher $F_{\text {prompt }}$ than expected WIMP signal [3]. 


\section{Dark matter search results}

The full event selection for this WIMP dark matter search results in a total background expectation of $0.62_{-0.28}^{+0.31}$ events in the region of interest, of which $0.49_{-0.26}^{+0.27}$ is the expectation from neck $\alpha$ events. No data event is observed in the region of interest from this total exposure of 758 tonne-days [3]. 90\% confidence upper limits are placed on the spin-independent WIMP-nucleon cross-section as a function of WIMP mass. For WIMP masses greater than $30 \mathrm{GeV} / \mathrm{c}^{2}$, this result is the most sensitive to date for a dark matter search experiment with LAr, as shown in Figure 3.
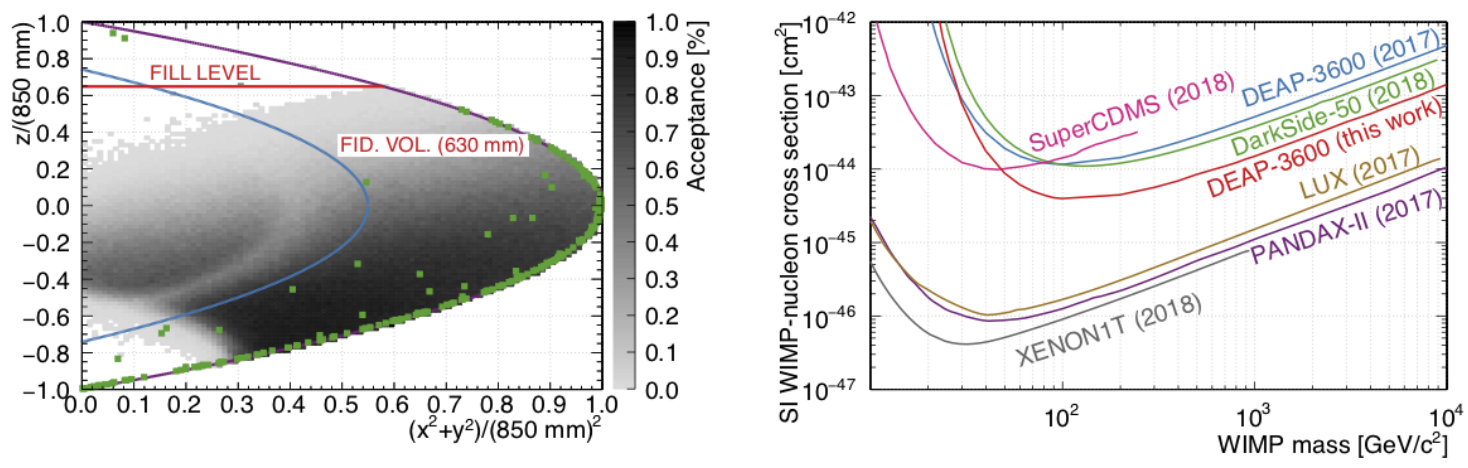

Figure 3: (Left) Reconstructed position of events passing the WIMP dark matter event selection other than the cut on reconstructed radius (green points). The acceptance for ${ }^{39} \mathrm{Ar} \beta$ decays prior to the PSD cut is shown in grey scale. Lines represent the inner AV surface, the LAr fill level, and the radial fiducial volume boundary. (Right) 90\% confidence upper limits on the spin-independent WIMP-nucleon cross-section as a function of WIMP mass, for a selection of direct detection experiments including this result. [3]

The above limits assume the Standard Halo Model (SHM). In [9], we have quantified the impact on the DEAP-3600 sensitivity of varying the dark matter velocity profile. For instance, if a significant fraction of dark matter travelled with a retrograde stellar stream (such as Koppelman 1) compared with the Solar System's orbit around the galactic centre, the speed and thus the kinetic energy of dark matter coming into Earth-based detectors would be greater. This would result in a higher fraction of interactions above threshold, implying a better detector sensitivity as shown in Figure 4. The reverse is true for prograde stellar streams, such as Nyx or the Gaia Sausage: under such dark matter velocity hypotheses, direct detection limits are slightly weaker than in the SHM.

Also shown in Figure 4 are results in the so-called xenonphobic coupling scenario, where WIMP-proton and WIMP-neutron interactions are such that the DEAP-3600 limits are worldleading at high WIMP mass. More DEAP-3600 limits on the coupling strength of different effective contact interaction operators, isovector couplings, as well as millicharge, magnetic dipole, electric dipole and anapole WIMP-nucleon interactions are also presented in [9].

\section{Conclusion and outlook}

DEAP-3600 is the most sensitive direct detection experiment looking for dark matter with LAr to date. In its first year of data corresponding to a total exposure of 758 tonne-days, no event was seen in the region of interest and limits were placed on WIMP-nucleon cross-sections. Work is ongoing 

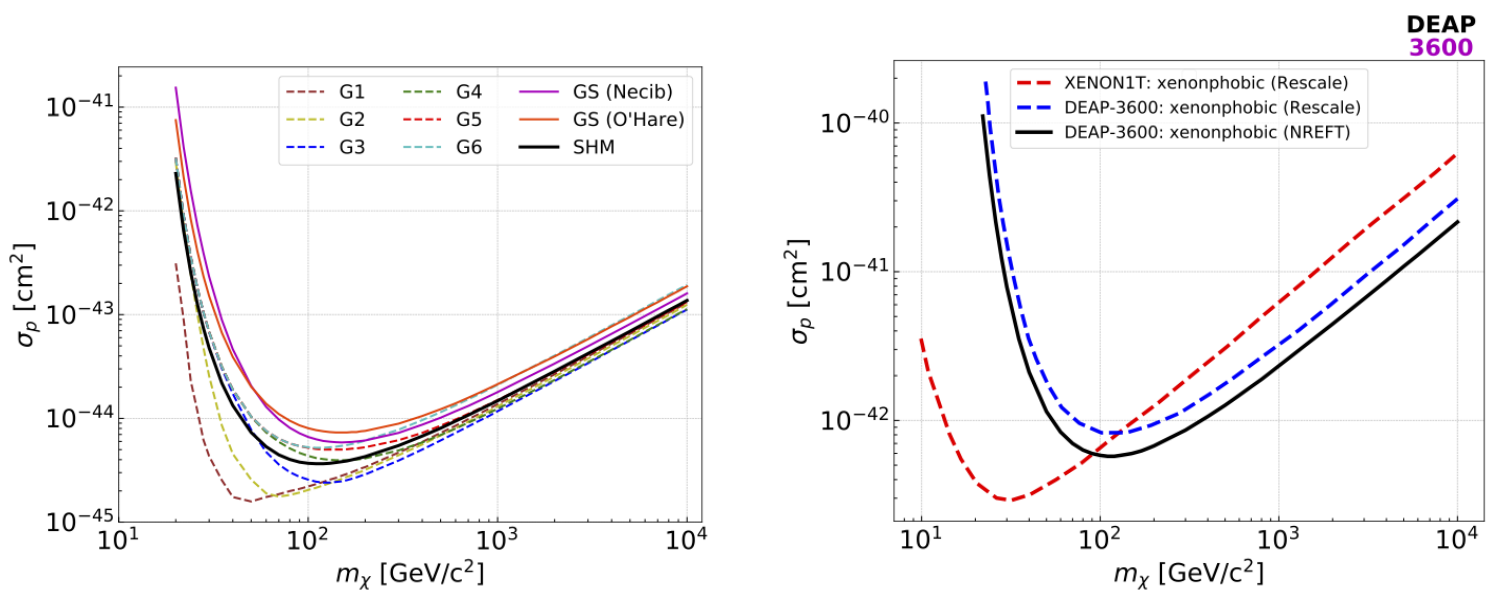

Figure 4: (Left) $90 \%$ confidence upper limits on the spin-independent WIMP-nucleon cross-section as a function of WIMP mass, for different possible halo substructures described in [9]. Limits on other effective operators describing WIMP-nucleon interactions are also shown there. (Right) Likewise 90\% upper limits in the xenonphobic coupling scenario. [9]

to carry out a multivariate analysis of the full second-fill dataset collected in 2016-2020, with a complete background model. Three machine-learning algorithms are trained against neck $\alpha$ events, to develop an event selection with improved signal acceptance and lower expected backgrounds.

Further, a hardware upgrade program is well underway, with the objective to mitigate limiting background sources in the experiment. Considered are a neck seal replacement that would allow a complete fill with LAr; a slow wavelength shifter coating on neck flow-guides, to remove neck backgrounds with PSD; an alternate cooling system, to avoid liquid-like scintillation in the neck; as well as necessary maintenance on cryogenic systems. The detector is now empty of LAr, still taking data with GAr and with vacuum, with and without calibration sources deployed. New dark matter search data in the upgraded DEAP-3600 experiment are expected in 2021.

DEAP collaboration members have joined the Global Argon Dark Matter Collaboration, with the objective to build DarkSide-20k, the next-generation liquid argon dark matter detector at LNGS. Design studies are starting for Argo, a multi-hundred tonnes detector with expected sensitivity surpassing the neutrino floor. The preferred site for Argo is SNOLAB.

\section{References}

[1] Planck Collaboration, Astronomy \& Astrophysics 641 A6 (2020). arXiv:1807.06209

[2] DEAP-3600 Collaboration, Astroparticle Physics 108, 1-23 (2019). arXiv:1712.01982

[3] DEAP-3600 Collaboration, Physical Review D 100, 022004 (2019). arXiv: 1902.04048

[4] DEAP-3600 Collaboration, Physical Review Letters 121, 071801 (2018). arXiv:1707.08042

[5] DEAP-3600 Collaboration, European Physics Journal C 80, 303 (2020). arXiv:2001.09855

[6] M. G. Boulay and A. Hime, Astroparticle Physics 25, 179 (2006).

[7] DEAP-3600 Collaboration, Physical Review D 100, 072009 (2019). arXiv: 1905.05811

[8] Y. Chen for the DEAP-3600 Collaboration, JINST 15, 05, C05061 (2020). arXiv:2004.02058

[9] DEAP-3600 Collaboration, Physical Review D 102, 082001 (2020). arXiv:2005.14667 